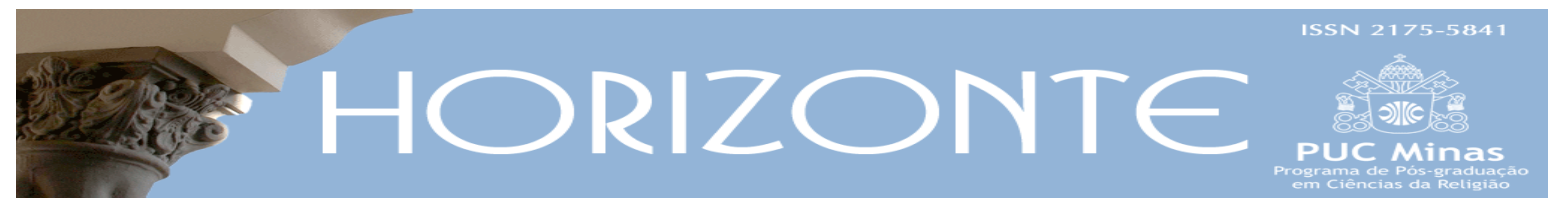

Nominata dos Avaliadores ad hoc 2012

(ف)

\title{
Nominata dos Avaliadores ad hoc 2012
}

1. Adilson José Francisco - UFMT

2. Adna Candido de Paula - Univ. Fed. dos Vales do Jequitinhonha e Mucuri

3. Adriano Correia Silva - UFG

4. Agemir Bavaresco - PUC RS

5. Agemir de Carvalho Dias - UFPR

6. Agenor Brighenti - PUC PR

7. Agnaldo Cuoco Portugal - UnB

8. Airton Luiz Jungblut - PUC RS

9. Albertino Luiz Gallina - UFSM

10. Alberto S. Moreira - PUC Goiás

11. Alessandro Manduco Coelho - UNINOVE, SP

12. Alexandre Antonio Cardoso - UFMG

13. Alzira Munhoz - ISTA/MG

14. Ana Raquel M. C. Portugal - UNESP/ Franca

15. Anaxsuell Fernando Silva - UNICAMP

16. Andrey Ivanov - UNESP

17. Anelito Pereira de Oliveira - UNIMONTES

18. Anita Martins Rodrigues de Moraes - UNICAMP

19. Antônio Alvimar Souza - UEMC/MG

20. Antonio Maximo von Sohsten Gomes Ferraz - UFPA

21. Antônio T. Oliveira Xavier - UCSAL

22. Antonio Vidal Nunes - UFES

23. Aparecida de Fátima Bueno - USP

24. Arnaldo Érico Huff Júnior - UFJF

25. Artur Cesar Isaia - UFSC 
26. Áurea Marin - ISTA/MG

27. Brenda Maribel Carranza Dávila - PUC Campinas

28. Breno Martins Campos - Mackenzie/SP

29. Cândido Moreira Rodrigues - UFMT

30. Carlos Alberto Steil - UFRGS

31. Carlos Eduardo Sell - UFSC

32. Carlos R. Caldas Filho - UMESP

33. Carolina Teles Lemos - PUC Goiás

34. Ceci M. B. Mariani - PUC Campinas

35. Cecília Cintra Cavaleiro de Macedo - UNIFESP

36. Cícero Cunha Bezerra - UFS

37. Cláudia Pellegrino Drucker - UFSC

38. Claudio de Oliveira Ribeiro - UMESP

39. Cláudio Vianney Malzoni - UNICAP

40. Cléber Eduardo dos Santos Dias - PUC RS

41. Daniel Alves - UFG

42. David Wilson - University of Edinburgh /Escócia

43. Denise dos Santos Rodrigues - UFRJ

44. Derivaldo dos Santos - UFRN

45. Donizete Rodrigues - UBI (Portugal) e Salamanca (Espanha)

46. Ednilson Turozi de Oliveira - FC - Faculdade Claretiana

47. Eduardo Gross - UFJF

48. Eduardo Guerreiro Brito Losso - UFRRJ

49. Eduardo Gusmão de Quadros - PUC Goiás

50. Elisa Rodrigues - UNICAMP

51. Ênio José da Costa Brito - PUC São Paulo

52. Ester Fraga Vilas-Bôas Carvalho do Nascimento - UNIT Aracajú/ SE

53. Esther Solano - UFU/MG

54. Eva Lenita Scheliga - UNIFESP

55. Faustino Teixeira - UFJF

56. Fernanda Lemos - UFPB 
57. Fernando Altmeyer Júnior - PUC SP

58. Flavio Augusto Senra Ribeiro - PUC MG

59. Flávio Munhoz Sofiati - UFG

60. Francisco das Chagas Albuquerque - FAJE

61. Frank Usarski - PUC- SP

62. Geraldo Caliman - UCB

63. Geraldo Luiz de Mori - FAJE

64. Gilberto Gonçalves Garcia - PUC Goiás

65. Gilbraz Aragão - UNICAMP

66. Gisela Isolde Waechter Streck - PUC RS

67. Gizele Zanotto - UPF/RS - Universidade Passo Fundo

68. Haroldo Reimeir - PUC Goiás

69. Haudrey Geminiani Calvelli - UFJF

70. Helder Games - USP

71. Helmut Renders - UMESP

72. Hilário Henrique Dick - UNISINOS

73. Irene Araújo van den Berg - UERN

74. Isidoro Mazzorollo - PUC Rio

75. Iuri Andréas Reblin - EST

76. Ivoni Richter Reimer - UFSC

77. Jaldemir Vitório - FAJE

78. Jenny González Muñoz - FBA/Venezuela

79. João Carlos Almeida - FD - Faculdade Dehoniana/SP

80. João Décio Passos - PUC-SP

81. Joe Marçal Gonçalves dos Santos - PUC Rio Grande do Sul

82. José Américo de Lacerda Júnior - UFF

83. José Bittencourt Filho - UNB

84. José Luiz Furtado - UFOP

85. José Maria da Silva - Faculdade Estácio de Sá-JF/MG

86. José Pedro Luchi - UFES

87. José Ternes - PUC Goiás

88. Karina Kosicki Bellotti - UFPR 
89. Laude Erande Brandenburg - EST

90. Leila Marrach Basto de Albuquerque - UE Júlio Mesquita/SP

91. Lemuel Dourado Guerra Sobrinho - UFPB

92. Leomar Antônio Brustolin - PUC RS

93. Lúcia Pedrosa Pádua - PUC Rio

94. Luciana Eleonora de Freitas Calado Deplagne - UFPB

95. Luiz Carlos Susin - PUC RS

96. Luís Filipe Estevinha Lourenço Rodrigues - CFUL

97. Luis Mauro Sá Martino - PUC SP

98. Luiza E. Tomita - UNISAL

99. Lurdes Caron - UNC/SC

100. Marcel de lima Santos - UFMG

101. Marcelo Kunrath Silva - UFRGS

102. Marcelo Martins Barreira - UFES

103. Márcio Fabri dos Anjos - Centro Universitário São Camilo/SP

104. Marcos Aparecido Lopes - UNICAMP

105. Marcos Orison Nunes de Almeida - FTSA/PR - Fac. Teol. Sul Americana/PR

106. Marcos Roberto Nunes Costa - UFPE

107. Maria Helena Nery Garcez - Inst. Intern. de Ciências Sociais - IICS/CEU

108. Maria Inês S. Borges - UFPR

109. Maria Severina Batista Guimarães - UEG

110. Maria Simone Marinho Nogueira - UEPB

111. Mariluze Ferreira de Andrade e Silva - UFSJ

112. Marina Bay Frydberg - UFRGS

113. Marlon Ronald Fluck - FEPAR/PR

114. Massimo Sciarretta - UNIRIO

115. Monica do Nascimento Figueiredo - UFRJ

116. Nilton José dos Anjos de Oliveira - UFRJ

117. Orivaldo Pimenta Lopes Júnior - UFRN

118. Osmar Pereira Oliva - UNIMONTES

119. Patrícia Carla de Melo Martins - CBM

120. Paulo Afonso Araújo - UFJF 
121. Paulo Jackson Nóbrega de Souza - FAJE

122. Paulo Rodrigues Romeiro - UPM - Mackenzie /SP

123. Paulo Sérgio Carrara - FAJE

124. Raymundo Heraldo Maués - UFMA

125. Ricardo Bitun - UPM - Mackenzie/SP

126. Robson de Medeiros Alves - PUC SP

127. Rodrigo Ribeiro Alves Neto - UFRJ

128. Ronaldo de Paula Cavalcante - UNIDA-ES

129. Rosa Gitana Krob Meneghetti - UNIMEP

130. Rudolf E. von Sinner - EST

131. Salma Ferraz - UFSC

132. Sandra Duarte de Souza - UMESP

133. Sávio Calos Desan Scopinho - FIC - Faculdades Integradas Claretianas/SP

134. Sérgio Junqueira - PUC PR

135. Sergio Néstor Osório García - Universidad Nueva Granada (Colômbia)

136. Sidney Antonio da Silva - UFAM

137. Sidney de Moraes Sanches - FTSA- Faculdade de Teologia Sul Americana/ PR

138. Sílvia Maria de Contaldo - PUC Minas

139. Sílvia Regina Alves Fernandes - UFRRJ

140. Steven Joseph Engler - Mount Royal University/Canadá

141. Suely Aparecida Martins - UEOPR

142. Valeriano dos Santos Costa - PUC SP

143. Valmor da Silva - PUC Goiás

144. Vitor Galdino Feller - ITESC

145. Waldecy Tenório de Lima - PUC SP

146. Waldir Souza - PUC PR

147. Walter Ferreira Salles - PUC Campinas

148. Wania Amélia Belchior Mesquita - UFRJ

149. Zuleica Dantas Pereira Campos - UNICAP

150. Zwinglio Mota Dias - UFJF 